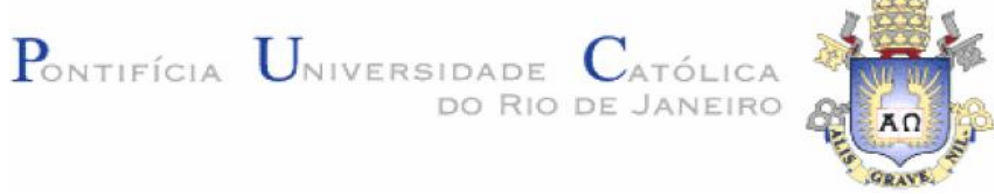

Carlos Fernando Cogollo Aponte

Utilização do Método dos Elementos Finitos no Desenvolvimento de Modelos de Corte de Material

Dissertação apresentada como requisito parcial para obtenção do título de Mestre pelo Programa de PósGraduação em Engenharia Civil da PUC-Rio.

Orientador: Prof. Sergio Augusto Barreto da Fontoura Co-orientador: Dr. Nelson Inoue 


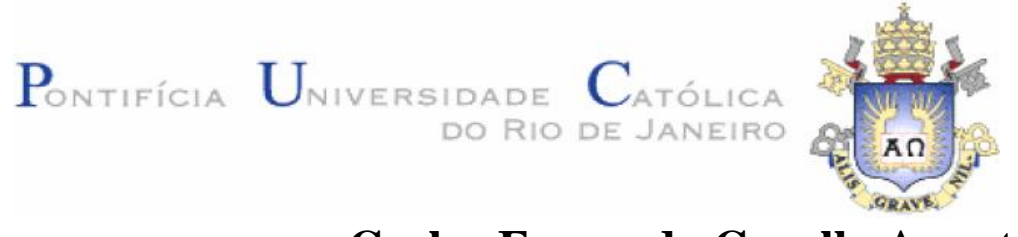

Carlos Fernando Cogollo Aponte

\section{Utilização do Método dos Elementos Finitos no Desenvolvimento \\ de Modelos de Corte de Material}

Dissertação apresentada como requisito parcial para obtenção do título de Mestre pelo Programa de Pós-Graduação em Engenharia Civil do Departamento de Engenharia Civil da PUC-Rio. Aprovada pela Comissão Examinadora abaixo assinada.

Prof. Sergio Augusto Barreto da Fontoura

Orientador PUC-Rio

Dr. Nelson Inoue

Co-orientador PUC-Rio

Prof. Celso Romanel

PUC-Rio

Prof. Tarcisio Celestino USP-São Carlos

Prof. José Eugênio Leal

Coordenador Setorial do Centro Técnico Científico - PUC-Rio 
Todos os direitos reservados. É proibida a reprodução total ou parcial do trabalho sem autorização da universidade, do autor e do orientador.

\section{Carlos Fernando Cogollo Aponte}

Graduou-se em Engenharia Civil na UIS Universidade Industrial de Santander Colômbia em 1999/2, possui especialização em GIS pela Universidade Distrital Francisco José de Caldas de Bogotá na Colômbia em 2005/2. Tem trabalhado em várias áreas da engenharia civil atuando agora no setor público. No ano 2009 ingressou ao curso de Mestrado em Engenharia Civil na Pontifícia Universidade Católica do Rio de Janeiro, na área de geotécnica, desenvolvendo dissertação de mestrado na linha de pesquisa Geomecânica do Petróleo.

Ficha Catalográfica

Cogollo A., Carlos Fernando.

Utilização do Método dos Elementos Finitos no Desenvolvimento de Modelos de Corte de Material / Carlos Fernando Cogollo Aponte; Orientador: Sergio A. B. da Fontoura; Co-orientador: Nelson Inoue. - Rio de Janeiro: PUC-Rio, Departamento de Engenharia Civil, 2011.

v., $101 \mathrm{f}:$ Il. ; $30 \mathrm{~cm}$

1. Dissertação (mestrado) - Pontifícia Universidade Católica do Rio de Janeiro, Departamento de Engenharia Civil.

Incluí referências bibliográficas.

1. Engenharia Civil - Teses. 2. Elementos finitos. 3. Corte de materiais. 4. Modelagem. 5. Forças. 6. MSE. 7. Torque. 8. Modelos Constitutivos. 9. Problemas dinâmicos. 10. Dependência da taxa. 11. Grandes deformações. 12. Problemas de Contato. 13. Mecanismos de falha. 
Al Señor Jesús mi Dios "al único y sabio Dios, nuestro Salvador, sea gloria y majestad, imperio y potencia, ahora y por todos los siglos" 


\section{Agradecimentos}

A meu Deus Jesus Cristo, por permitir tudo e me segurar em seus propósitos até o fim, muito obrigado.

A Nohora Isabel minha amada esposa e minhas filhas Isabella e Gabriela por seu grande amor.

Agradeço a toda minha família, mãe, irmãos, sobrinhos, irmãos, tios, sogros e primos pelo grande apoio oferecido.

A Igreja do Senhor na Colômbia IPUC Bucaramanga Barrio Mutis e a IPUB em São João de Meriti RJ.

Ao Professor Sergio Fontoura pela orientação ao longo da pesquisa, pelo apoio, paciência e seu conhecimento.

Obrigado Nelson Inoue por sua grande ajuda dedicação e amizade ao longo dos anos de trabalho no GTEP.

A PUC-Rio, seus funcionários e as agências CNPq (Conselho Nacional de Desenvolvimento Científico e Tecnológico) e CAPES (Coordenação de Aperfeiçoamento de Pessoal de nível superior) pela oportunidade oferecida e pela ajuda econômica.

Agradeço a Contraloría General de la República de Colombia pelo apoio e a oportunidade oferecida.

Agradeço a meus amigos do Mestrado pela amizade e companhia, a todos os colombianos que estão na PUC-Rio e foram meus amigos e família, a os meninos da casa, muito obrigado.

Agradeço a os colegas do GTEP, os funcionário e amigos que me ofereceram sua amizade e ajuda. Em fim a cada uma das pessoas que me apoiaram muito obrigado. 


\section{Resumo}

Aponte, Carlos Cogollo; Fontura, Sergio Augusto Barreto. Utilização do Método dos Elementos Finitos no Desenvolvimento de Modelos de Corte de Material. Rio de Janeiro, 2011. 101p. Dissertação de Mestrado Departamento de Engenharia Civil, Pontifícia Universidade Católica do Rio de Janeiro.

Entre os vários processos envolvidos na produção de petróleo e gás, o processo de perfuração tem recebido grande atenção recentemente. Pesquisas estão sendo direcionadas para este tema com o objetivo de melhorar o desempenho das brocas de perfuração. Assim, o interesse da indústria do petróleo é aumentar a taxa de penetração, reduzir o tempo de manobras, aumentar a vida útil das brocas e consequentemente reduzir gastos durante o processo de perfuração. Para alcançar estes objetivos, tradicionalmente, são empregados métodos de otimização de perfuração baseados em energia mecânica específica. Estes métodos utilizam como dados de entrada o peso sobre a broca, taxa de penetração, torque e resistência à compressão da rocha. Os métodos de otimização de perfuração ainda apresentam algumas dificuldades, tais como: relacionar energia mecânica específica com resistência à compressão simples e os métodos dependem de dados de perfuração de poços, como listados acima. Este trabalho tem como objetivo apresentar um estudo alternativo do processo de corte em rocha baseado em simulação numérica, especificamente utilizando um programa comercial de elementos finitos chamado Abaqus. A primeira parte do trabalhou consistiu no desenvolvimento de modelos de corte em metais baseado na lei de plasticidade de Johnson-Cook. A motivação na escolha destes modelos foi a grande quantidade de trabalhos encontrados na literatura que tratam do processo de usinagem em metais. Posteriormente, a segunda parte do trabalho, que trata do corte em rocha, foi conduzida. Modelos tridimensionais de ensaios de single cutter foram elaborados e simulados utilizando a lei de plasticidade de Drucker-Prager, adequada para rochas, que considera o efeito da pressão de confinamento.

\section{Palavras-chave}

Método dos elementos finitos; Processo de corte em metais e rocha; Leis de plasticidade de Johnson-Cook e Drucker-Prager. 


\begin{abstract}
Aponte, Carlos Cogollo; Fontura, Sergio Augusto Barreto (Advisor). Using Finite Element Model in the Development of Cutting Material Models. Rio de Janeiro, 2011. 101p. M.Sc. Dissertation -Departamento de Engenharia Civil, Pontifícia Universidade Católica do Rio de Janeiro.
\end{abstract}

Among the various processes involved in producing oil and gas, the drilling process has received great attention recently. Research is being directed to this issue in order to improve the performance of drill bits. Thus, the interest of the oil industry is to increase the penetration rate, reduce the time of switching, extending the life of drills and consequently reduce spending during the drilling process. To achieve these goals are traditionally used for drilling optimization methods based on mechanical specific energy. These methods use as input the weight on the drill penetration rate, torque and compressive strength of rock. The drilling optimization methods still present some difficulties, such as mechanical specific energy relationship with compressive strength and the methods depend on datadrilling, as listed above. This dissertation aims to present a study of alternative rock cutting process based on numerical simulation, specifically using a commercial finite element program called Abaqus. The first part of the work was the development of models of metal cutting based on the plasticity law of Johnson-Cook. The motivation in choosing these models was the large number of studies in the literature dealing with the machining process in metals. Subsequently, the second part of the work, which is cut in rock, was conducted. Three-dimensional models of single cutter tests were designed and simulated using the plasticity law of Drucker-Prager, suitable for rocks, which considers the effect of confining pressure.

\title{
Keywords
}

Finite element method; Procedure for cutting metal and rock; Plasticity laws of Johnson-Cook and Drucker-Prager. 


\section{Sumário}

1 Introdução $\quad 15$

$\begin{array}{ll}1.1 & \text { Objetivo do Trabalho. } \\ & 16\end{array}$

$\begin{array}{lll}1.2 & \text { Escopo do trabalho } & 17\end{array}$

2 Revisão Bibliográfica $\quad 18$

2.1 Simulação de corte em metais através do método dos elementos finitos: 18

2.1.1 Simulação com elementos finitos do processo de formação de cavacos na usinagem de aço ISI 1045 considerando alta velocidade 18

2.1.2 Estudos do efeito termo-mecânico na formação de chips durante $\begin{array}{ll}\text { o processo de usinagem } & 19\end{array}$

2.1.3 Simulação da usinagem de peças endurecidas 21

2.2 Simulação de corte em rochas através do método dos elementos finitos 22

2.2.1 Simulação numérica do corte e da geração de calor durante corte

linear em rocha 22

2.2.2 Simulação numérica de corte em rocha utilizando o programa

LS-DYNA 24

2.2.3 Simulação numérica de perfuração e corte em rocha. 25

3 Modelagem com o método dos elementos finitos (MEF) 26

3.1 Detalhamento das variáveis envolvidas no processo de corte 27

3.2 Método de solução das equações e não linearidade 28

3.3 Descrição do movimento 28

3.3.1 Formulação Lagrangiana 29

3.3.2 Formulação Euleriana 30

3.3.3 Formulação ALE (Arbitraria Lagrangiana Euleriana): 31

3.4 Problema do contato 32

3.5 Modelos constitutivos utilizados e lei de dano 34

3.5.1 Modelo de Johnson \& Cook 35

3.5.2 Modelo de Drucker-Prager 37

3.6 Modelos de dano e critérios de dano no modelo constitutivo de

Johnson \& Cook e de Drucker-Prager 44 
3.6.1 Modelos de ruptura dinâmico 45

3.6.2 Modelos de dano progressiva 46

4 Simulação do processo de corte pelo método dos elementos finitos 51

4.1 Validação das simulações realizadas $\quad 52$

4.2 Modelos de corte 2D em metais 52

4.2.1 Forças de corte e MSE nos modelos 2D em metais 55

4.2.1.1 Modelo GTEP9_2-00_05AtritoA: 55

4.2.1.2 Modelo GTEP9_2-00_05AtritoTermico: 56

4.2.1.3 Modelos GTEP9_2-00_05AtritoAERODEa, GTEP9_2-

00_05AtritoAERODEb1, GTEP9_2-00_05AtritoAERODEbOriginl: 57

4.2.1.4 Comparação dos resultados de corte em metais 2D 59

4.3 Simulação do corte em rocha através do modelo de plasticidade de

$\begin{array}{ll}\text { Drucker-Prager } & 61\end{array}$

4.3.1 Forças e energia (MSE) nos modelos 2D (SC_2D1, SC_2D3) e 3D

(SC_Cir10, SC_Cir11): 70

4.3.2 Forças, e MSE dos modelos 2D com dano Progressivo parâmetro

1E-18 (SC_2D1_0, SC_2D1_10, SC_2D3_0,SC_2D3_10): 70

4.3.3 Comparação das forças e MSE dos modelos 2D com dano Progressivo parâmetro 1E-18 (SC_2D1_0, SC_2D1_10, SC_2D3_0, SC_2D3_10): 78

4.3.4 Forças, e MSE dos modelos 3D com dano Progressivo parâmetro

1E-18 (SC_Cir10_0, SC_Cir10_10,SC_Cir11_0,SC_Cir11_10):

4.3.5 Comparação das forças e MSE dos modelos 3D com dano

Progressivo parâmetro 1E-18 (SC_Cir10_0, SC_Cir10_10, SC_Cir11_0, SC_Cir11_10):

5 Conclusões e Sugestões para trabalhos futuros: 


\section{Lista de figuras}

Figura 2.1. Malha de elementos finitos utilizada para discretizar o problema de corte 19

Figura 2.2. Malha utilizada para a simulação de corte 20

Figura 2.3. Variação da magnitude da força de corte 21

Figura 2.4. Malha e condições de contorno do modelo 21

Figura 2.5. Malha de elementos finitos para (a) chip contínuo e (b) chip segmentado. Do lado direito da malha (b) está uma fotomicrografia de uma secção de um chip real. (Eu-Gene \& Aspinwall, 2002). 22

Figura 2.6. Modelo 2D de corte linear (Mishra \& Khair, 2005). 23

Figura 2.7. Modelo 3D de corte linear (Mishra \& Khair, 2005). 23

Figura 2.8. Geometria do Modelo. (Tulu et al. 2008) 25

Figura 3.1. Principais variáveis envolvidas no processo de simulação de corte utilizando o programa Abaqus.

Figura 3.2. Exemplo do comportamento da malha na formulação Lagrangiana J.

Donea et al. (Encyclopedia of Computational Mechanics, Vol. 1)

Figura 3.3. Exemplo do comportamento da malha na formulação Euleriana J.

Donea et al (Encyclopedia of Computational Mechanics, Vol. 1) 30

Figura 3.4. O operador ALE. (Shekhar, 2009). 32

Figura 3.5. Modelo de superfícies fixas que apresenta as duas superfícies de contato pré-definidas durante o processo de corte.

Figura 3.6. Modelo com erosão que apresenta a superfície da ferramenta e o

domínio da peça, onde superfícies de contato são criadas na face livre.

Figura 3.7. Topologia de uma superfície de contato do tipo erosão (Abaqus, 2010). 34

Figura 3.8. Modelo Linear Drucker-Prager (Abaqus, 2010). 39

Figura 3.9. Modelo hiperbólico Drucker-Prager (ABAQUS, 2010). 41

Figura 3.10 Modelo exponencial Drucker-Prager (ABAQUS, 2010). 41

Figura 3.11 Diferentes definições da evolução do dano com base no deslocamento plástico: (a) tabular, (b) linear e (c) exponencial (ABAQUS, 2010).

Figura 3.12. Curva tensão deformação representando o modelo de ruptura dinâmico (vermelho) e o modelo de dano progressivo. (ABAQUS, 2010 modificado).

Figura 4.1. Geometria dos modelos de corte em metais 2D (Dimensões em m). 52 
Figura 4.2. Força de corte (a) no modelo de "superfícies fixas" (b) modelo com erosão.

Figura 4.3. Variação das tensões de Von Misses em um modelo com "superfícies fixas".

Figura 4.4. Variação da força de corte com o deslocamento do cortador. $\quad 56$

Figura 4.5. Geometria do chip devido o efeito da temperatura. 56

Figura 4.6. Efeito da temperatura na variação da força de corte com o deslocamento do cortador.

Figura 4.7. (a) GTEP9_2-00_05AtritoAERODEa, (b) GTEP9_2-

00_05AtritoAERODEb1, (d) GTEP9_2-00_05AtritoAERODEbOriginl

Figura 4.8. Forças vs. deslocamentos (a) GTEP9_2-00_05AtritoAERODEa,

(b) GTEP9_2-00_05AtritoAERODEb1, (c) GTEP9_2-

00_05AtritoAERODEbOriginl

Figura 4.9. Variação da força de corte com o deslocamento do cortador para os cinco modelos.

Figura 4.10. Resultados de força para os cinco modelos.

Figura 4.11. Resultados de MSE para os cinco modelos.

Figura 4.12. Curvas tensão-deformação obtidas de ensaios numéricos no programa PFC2D para o Mármore de Cartago para diferentes pressões de confinamento.

Figura 4.13. Dados obtidos para calibração da lei endurecimento da lei de Drucker-Prager para o Mármore de Cartago.

Figura 4.14. Desenvolvimento da lei de Drucker-Prager. (a) Malha de elementos finitos para discretizar a amostra de rocha, (b) Gráfico p-q com as superfícies de plastificação experimental e ajustada pelo programa Abaqus e (c) Curvas tensão-deformação utilizadas para obter os valores de deformação plástica equivalente no início do dano.

Figura 4.15. Malha grossa de elementos finitos para os modelos SC_2D1_ (0 e $10 \mathrm{MPa})$.

Figura 4.16. Malha refinada de elementos finitos para os modelos SC_2D3 _(0 e 10MPa)

Figura 4.17. Malha grossa de elementos finitos utilizada nos modelos $\mathrm{SC}_{-}$ Cir10_(0 - 10) MPa 
Figura 4.18 Malha "fina" de elementos finitos utilizada nos modelos SC_ Cir11_(0 - 10) MPa.

Figura 4.19. Diferentes estágios do modelo 2D SC_2D1_0 com malha grossa e sem pressão de confinamento.

Figura 4.20. Forças de corte sem pressão de confinamento na direção:

(a) horizontal e (b) vertical.

Figura 4.21. Diferentes estágios do modelo 2D SC_2D1_10 com malha grossa e pressão de confinamento de $10 \mathrm{MPa}$.

Figura 4.22. Forças de corte com pressão de confinamento na direção: (a) horizontal e (b) vertical.

Figura 4.23. Diferentes estágios do modelo SC_2D3_0 com malha refinada e sem pressão de confinamento.

Figura 4.24. Forças de corte sem pressão de confinamento na direção: (a) horizontal e (b) vertical.

Figura 4.25. Diferentes estágios do modelo SC_2D3_10 com malha refinada e pressão de confinamento de $10 \mathrm{MPa}$.

Figura 4.26. Forças de corte com pressão de confinamento na direção: (a) horizontal e (b) vertical.

Figura 4.27. Comparação das forças de corte horizontal para modelos com e sem pressão de confinamento.

Figura 4.28. Comparação das forças de corte vertical para modelos com e sem pressão de confinamento.

Figura 4.29. Comparação da MSE dos modelos SC_2D1_0 (0 MPa) e SC_2D1_ $10(10 \mathrm{MPa})$ para a malha grossa.

Figura 4.30 Comparação das forças de corte horizontal para modelos com e sem pressão de confinamento.

Figura 4.31. Comparação das forças de corte vertical para modelos com e sem pressão de confinamento.

Figura 4.32. Comparação da MSE dos modelos SC_2D1_0 (0 MPa) e SC_ 2D1_10 (10 MPa) malha "refinada".

Figura 4.33. Comparação das forças de corte vertical para modelos com e sem pressão de confinamento (a) escala original e (b) escala ampliada.

Figura 4.34. Comparação da MSE dos modelos SC_2D1 (0 e 10 MPa malha 
grossa) e SC_2D3 (0 e $10 \mathrm{MPa}$ malha refinada).

Figura 4.35. Diferentes estágios do modelo 3D SC_Cir10_0 com malha grossa e pressão de confinamento de $0 \mathrm{MPa}$.

Figura 4.36. Força de corte na direção: (a) horizontal, (b) vertical e (c) ampliação do gráfico da força de corte na direção vertical.

Figura 4.37. Diferentes estágios do modelo 3D SC_Cir10_10 com malha grossa e pressão de confinamento de $10 \mathrm{MPa}$.

Figura 4.38 Força de corte na direção: (a) horizontal, (b) vertical e (c)

ampliação do gráfico da força de corte na direção vertical.

Figura 4.39. Diferentes estágios do modelo SC_Cir11_0 com malha refinada e pressão de confinamento de $0 \mathrm{MPa}$.

Figura 4.40 Força na direção: (a) horizontal e (b) vertical no modelo com malha refinada SC_Cir11_0 sem pressão de confinamento.

Figura 4.41. Diferentes estágios do modelo SC_Cir11_10 com malha refinada e pressão de confinamento de $10 \mathrm{MPa}$.

Figura 4.42. Força na direção: (a) horizontal e (b) vertical no modelo com malha refinada SC_Cir11_0 com pressão de confinamento de $10 \mathrm{MPa}$.

Figura 4.43. Comparação das forças de corte horizontal para modelos com e sem pressão de confinamento.

Figura 4.44. Comparação das forças de corte horizontal para modelos com e sem pressão de confinamento (a) escala original e (b) escala ampliada. Figura 4.45. Comparação de MSE dos modelos SC_Cir10_0 (0 MPa) e SC_Cir10_10 (10 MPa) malha grossa.

Figura 4.46. Comparação das forças de corte horizontal para modelos com e sem pressão de confinamento.

Figura 4.47. Comparação das forças de corte vertical para modelos com e sem pressão de confinamento (a) escala original e (b) escala ampliada.

Figura 4.48. Comparação da MSE dos modelos SC_Cir11_0 (0 MPa) e SC_Cir11_10(10 MPa) malha "refinada".

Figura 4.49. Comparação das forças na direção horizontal dos modelos

SC_Cir10 (0 e $10 \mathrm{MPa})$ e SC_Cir11 (0 e $10 \mathrm{MPa})$.

Figura 4.50. Comparação de MSE dos modelos SC_Cir10 (0 e $10 \mathrm{MPa})$ e SC_Cir11 (0 e $10 \mathrm{MPa})$. 


\section{Lista de tabelas}

Figura 4.1. Propriedades geométricas dos modelos 2D. 53

Figura 4.2. Parâmetros utilizados na simulação de corte em metais. 53

Figura 4.3. Resumo das características dos modelos 2D para corte em metais 54

Figura 4.4. MSE para os modelos GTEP9_2-00_05AtritoAERODEa,

GTEP9_2-00_05AtritoAERODEb1, GTEP9_2-00_05AtritoAERODEbOriginl. 59

Figura 4.5. Dados da geometria dos modelos SC_2D. 67

Figura 4.6. Parâmetros da geometria dos modelos SC_Cir. 69

Figura 4.7. Principais características geométricas e parâmetros dos modelos

2D e 3D para o corte em rocha com pressão de confinamento de 0 e $10 \mathrm{MPa} .69$ 